Meeting report

\title{
Cell contact interactions in rheumatology, The Kennedy Institute for Rheumatology, London, UK, 1-2 June 2000 Danielle Burger
}

Clinical Immunology Unit, University Hospital, Geneva, Switzerland

Received: 13 July 2000

Accepted: 14 August 2000

Published: 30 August 2000
Arthritis Res 2000, 2:472-476

(c) Current Science Ltd (Print ISSN 1465-9905; Online ISSN 1465-9913)

\begin{abstract}
The intricate interactions that regulate relationships between endogenous tissue cells and infiltrating immune cells in the rheumatic joint, particularly in rheumatoid arthritis (RA), were the subject of the meeting. A better understanding of these interactions might help to define intervention points that could be used to develop specific therapies. The presentations and discussions highlighted the fact that, once chronic inflammation is established, several proinflammatory loops involving tumour necrosis factor (TNF)- $\alpha$ and interleukin (IL)-1 $\beta$ can be defined. Direct cellular contact with stimulated T lymphocytes induces TNF- $\alpha$ and IL-1 $\beta$ in monocytes which in turn induce functions in fibroblast-like synoviocytes. The latter include the production of stromal cell-derived factor- $1 \alpha$ (SDF-1 $\alpha$ ) which enhances the expression of CD40L in Tcells, which stimulates SDF- $1 \alpha$ production in synoviocytes, which in turn protects $T$ and $B$ cells from apoptosis and enhances cell recruitment thus favoring inflammatory processes. IL-1 $\beta$ and TNF- $\alpha$ also induce IL-15 in fibroblast-like synoviocytes, which induces the production of IL-17 which in turn potentiates IL-1 $\beta$ and TNF- $\alpha$ production in monocyte-macrophages. This underlines the importance of TNF- $\alpha$ and IL-1 $\beta$ in RA pathogenesis, and helps explain the efficiency of agents blocking the activity of these cytokines in RA. Factors able to block the induction of cytokine production (such as apolipoprotein $A-I$ [apo A-I] and interferon [IFN]- $\beta$ ) might interfere more distally in the inflammatory process. Furthermore, stimulated $T$ lymphocytes produce osteoclast differentiation factor (ODF), which triggers erosive functions of osteoclasts. Therefore, factors capable of affecting the level of T lymphocyte activation, such as IFN- $\beta$, IL-15 antagonist, or SDF- $1 \alpha$ antagonist, might be of interest in RA therapy.
\end{abstract}

Keywords: cell-cell interactions, cytokines, inflammation, rheumatoid arthritis

\section{Introduction}

The inflammatory site is the locus of many cellular interactions between specific tissue cells and infiltrating cells of the immune system. In RA many cell types participate in the pathogenic mechanisms that finally lead to tissue destruction (cartilage digestion and bone erosion). Among

apo A-I = apolipoprotein-AI; DC = dendritic cell; HDL = high-density lipoprotein; IFN = interferon; IL = interleukin; MIP = macrophage inflammatory protein; MMP = metalloproteinase; ODF = osteoclast differentiation factor; OPGL = osteoprotegerin ligand; PI3-K = phosphatidylinositol 3-kinase; $\mathrm{RA}=$ rheumatoid arthritis; SDF- $1 \alpha=$ stromal cell-derived factor- $1 \alpha$; SLE $=$ systemic lupus erythematosus; TIMP $=$ tissue inhibitor of metalloproteinase; TNF = tumour necrosis factor; WAS $=$ Wiskott-Aldrich syndrome. 
these cells $\mathrm{T}$ lymphocytes may play an important part, being the most abundant infiltrating cells in the pannus. Although $\mathrm{T}$ lymphocytes are claimed to participate in RA pathogenesis, knowledge of the mechanisms by which they exert their pathogenic effect(s) is still elusive. Monocyte-macrophages, the other main type of infiltrating cell in the pannus, play a crucial role because one of their main functions is to release various pro-inflammatory cytokines, including TNF- $\alpha, \mathrm{IL}-1 \beta$, and IL-17, which participate in the induction of metalloproteinase (MMP) secretion by stromal cells (fibroblast-like synoviocytes) and osteoprotegerin ligand (OPGL) expression in osteoblasts. MMPs participate in cartilage digestion and OPGL induces maturation of osteoclasts, which are involved in bone resorption. The meeting was held to highlight some recent key advances in the intricate interactions between these cells. It was coorganized by Drs M Feldmann and F Brennan (Kennedy Institute for Rheumatology, London, UK) and Drs J-M Dayer and D Burger (Division of Immunology and Allergy, University Hospital, Geneva, Switzerland); 65 researchers from eight countries attended the meeting at the Kennedy Institute for Rheumatology. Three half-day sessions were organized, each with three main lectures and up to five short communications followed by a general discussion.

\section{T cell-macrophage interactions}

The subject was introduced by Dr B Bresnihan (Department of Rheumatology, St Vincent's Hospital, Dublin, Ireland), who, as a clinical rheumatologist, pinpointed the relevance of $\mathrm{T}$ lymphocyte-monocyte-macrophage contact in RA pathogenesis. As less invasive biopsies are now possible, synovial tissue samples are more accessible, allowing direct analysis of cell populations and comparison between cell infiltrate and clinical status. The cellular composition of infiltrates varies from one patient to another. In general, the number of T lymphocytes in follicular structures (which resemble germinal centers) around blood vessels similar to high endothelial venules augments with time and correlates with disease severity. Monocytemacrophages migrate from the vessel through the follicular structure to the more superficial layer of the pannus lining-layer, becoming more and more activated (as revealed by CD68 expression). The radiological outcome of patients correlates with the number of macrophages in the synovium. These histological and clinical results pinpoint the importance of $\mathrm{T}$ lymphocyte and monocytemacrophage infiltrates in terms of cellular composition and interaction in disease progression and severity.

T lymphocytes are currently thought to play a pivotal part in the pathogenesis of RA (although this is controversial [1]). However, knowledge of the mechanism(s) by which they exert their pathogenic effect remains elusive. Contact-mediated signaling of monocytes by stimulated $\mathrm{T}$ cells is a potent proinflammatory mechanism that triggers massive upregulation of the proinflammatory cytokines IL-1 and TNF- $\alpha$. These cytokines play important roles in RA and their blockade is an efficient way to diminish symptoms in patients. Since the contact-mediated induction of cytokine production seems to play a major part in RA pathogenesis, the modulation of this mechanism is of great therapeutic interest.

On the basis of the observation that autoreactive $\mathrm{T}$ lymphocytes are present in the bloodstream of RA patients and normal subjects, Dr D Burger (Division of Immunology and Allergy, University Hospital, Geneva, Switzerland) hypothesized that factors aimed at inhibiting monocyte activation ought to be present in plasma/serum. We demonstrated that contact induction of IL-1 $\beta$ and TNF- $\alpha$ was inhibited by human adult serum but not by serum from human umbilical cord or fetal calves. The serum inhibitory factor was identified as apo A-I, a negative acute-phase protein associated with high-density lipoproteins (HDL). Although apo A-I binds to stimulated T cells, the interaction of the inhibitory factor with surface molecules involved in contact activation of monocyte-macrophages (CD69, $\beta 2$-integrins and membrane-associated TNF- $\alpha$ ) remains to be demonstrated. In contrast with its action in normal synovial tissue, apo A-I infuses into the RA synovium, the immunostaining being particularly intensive in the perivascular regions. These results reveal a novel anti-inflammatory function of apo A-I that might lead to new therapeutic approaches in chronic inflammatory diseases such as RA.

Dr F Brennan (Kennedy Institute of Rheumatology, London, UK) demonstrated the importance of cell contact for TNF- $\alpha$ production by cellular extracts of RA synovial tissue. In contrast with whole extracts, cellular extracts that are depleted of $T$ cells do not produce TNF- $\alpha$, whereas TNF- $\alpha$ production is restored by fixed, RA T cells. RA T cells display the activation phenotype, with more than $85 \%$ of them expressing CD45RO. Depending on the T-cell stimulus, different signaling pathways are induced in monocyte-macrophages leading to TNF- $\alpha$ production. Indeed, activation of $\mathrm{T}$ cells by a cytokine cocktail (IL-15 or IL-2, IL-6 and TNF) or anti-CD3 antibodies leads to TNF- $\alpha$ induction in monocyte-macrophages involving activation of NFKB and phosphatidylinositol 3-kinase (PI3-K), respectively; the engagement of CD45 in monocytes induces TNF- $\alpha$ through both NFKB and PI3-K pathways. In monocytes overexpressing $I \kappa B$ (as a result of adenoviral gene transfer), TNF- $\alpha$ induction by RA T cells has been shown to be NFKB dependent, whereas TNF- $\alpha$ production triggered by anti-CD3-stimulated $\mathrm{T}$ lymphocytes was NFKB independent and mainly PI3-K-dependent. Interestingly, the inhibition of the PI3-K pathway by wortmannin in RA synovial cells resulted in the overproduction of TNF- $\alpha$. Together these results suggest that TNF- $\alpha$ induced through the activation of NFKB promotes pathogenic inflammatory functions that escape the regulatory mechanisms controlled by other signaling pathways. 
Therefore, targeting of NFKB in chronic inflammation might be a promising therapeutic approach. By using monocytemacrophages overexpressing IKB (transferred by adenovirus), a group at the Kennedy Institute of Rheumatology (Drs M Feldmann, C Ciesielski and A Foey) showed that $\mathrm{NFKB}$ is involved in the induction of macrophage inflammatory protein (MIP)- $1 \alpha, \mathrm{IL}-6, \mathrm{MMP}-1$, and MMP-3, to a lesser extent in the induction of IL-1 $\beta$ and IL-8, but not in the induction of IL-10, IL-1Ra, IL-11, and tissue inhibitor of metalloproteinase (TIMP)-1; IL-10 is regulated through the PI3-K pathway upon induction by CD40 engagement.

Dr J-M Dayer (Division of Immunology and Allergy, University Hospital, Geneva, Switzerland) reported that IFN- $\beta$, which has been approved in multiple sclerosis therapy, also displays beneficial effects in RA. These in vivo effects correlate with the modulation of the contact-mediated induction of cytokine production in monocyte-macrophages in which IFN- $\beta$ inhibits TNF- $\alpha$ and IL- $1 \beta$ production and enhances IL-1Ra (IL-1 receptor antagonist) production. This strengthens the beneficial effect of IFN- $\beta$ and also suggests that stromal cells which produce IFN- $\beta$ might exert a feedback regulation of cytokine production.

Dr C Chizzolini (Division of Immunology and Allergy, University Hospital, Geneva, Switzerland) reported that polarized $T$ cells (Th1 or Th2) differentially affected the production of MMP-1 and MMP-9 in macrophages. Interestingly, membrane-associated IL-4 and TNF- $\alpha$ in Th2 cells concurrently induce MMP-1. Although IL-4 has no effect on freshly isolated peripheral blood mononuclear cells (PBMCs), it enhances T-cell-contact-mediated MMP1 production in monocytes cultured for 7 days with GMCSF while it inhibits MMP-9 production. This demonstrates that in some circumstances, IL-4 might exacerbate tissue destruction.

The general discussion highlighted the fact that, in vitro, the type of T-cell stimulus affects the type of products induced in monocyte-macrophages, suggesting that different cell surface molecules (which are differentially induced) in turn trigger different signalling pathways (eg $\mathrm{NFKB}$ or PI3-K) in monocyte-macrophages.

\section{Other immune interactions}

T cells that accumulate in the RA synovium express cytokines and activation markers that might be related to pathogenesis. Dr P Lipsky (NIAMS/NIH, Bethesda, MD, USA) showed that CD4+ T cells isolated from synovial tissue of RA patients express different patterns of cytokines and markers as assayed by single cell RT-PCR [2]. Noticeably, IL-2, IL-4 and IL-6 are not detected. No correlation has been found for the expression of cytokines, with the exception of correlations between expression of ODF (also called RANKL, TRANCE, and OPGL) and IL-10, and between ODF and TNF- $\alpha$. There is also a corre- lation between the expression of IL-10 and CCR7, ODF and CXCR4, LT- $\alpha$ and CCR6 and IFN- $\gamma$ and CCR5. Interestingly, CXCR4, which is usually a marker of naive T cells, is present in $76 \%$ of synovial CD4+ T cells. This high level of expression might be due to IL-15, which has been shown to increase the expression of CXCR4 and the migration of $T$ cells into the tissue. In synovial tissue SDF- $1 \alpha$ enhances the expression of CD4OL in T cells, which in turn stimulates SDF- $1 \alpha$ production in synoviocytes, which in turn protects $\mathrm{T}$ and $\mathrm{B}$ cells from apoptosis and enhances cell migration. This could induce a selfmaintaining proinflammatory loop, which might be important in RA pathogenesis.

IL-17 (which is produced by memory T cells) is present in both serum and synovial fluid of RA patients, where IL-15 is also expressed. Dr W Malinski (Department of Pathophysiology and Immunology, Institute of Rheumatology, Warsaw, Poland) demonstrated that IL-15 but not LPS (lipopolysaccharide) induces IL-17 production in PBMCs. IL-17 production is inhibited by both cyclosporin $A$ and methylprednisolone. In RA synovium, both the membraneassociated and soluble form of $\mathrm{IL}-15$ are expressed, mainly in fibroblast-like synoviocytes of the lining layer. Both IL-1 $\beta$ and TNF- $\alpha$ induce IL-15 in fibroblast-like synoviocytes; this in turn induces the production of IL-17 in T cells which potentiates IL- $1 \beta$ and TNF- $\alpha$ production in monocyte-macrophages. Here again there is a potential self-maintaining proinflammatory loop. The latter could be interrupted by IL-15 mutant/Fc $\gamma 2 \mathrm{a}$, an IL-15 antagonist which prevents collagen-induced arthritis in mice. Furthermore, IL-18 could be involved in this 'IL-15 loop', since it amplifies TNF- $\alpha$ production in monocyte-macrophages activated by contact with IL-15-stimulated $T$ cells ( $\mathrm{Dr}$ I Mclnnes, Centre for Rheumatic Diseases, Glasgow Royal Infirmary, Glasgow, UK).

The question of a function for dendritic cells (DCs) in RA was addressed by Dr D Katz (Department of Immunology, University College London Medical School, London, UK). In RA, DCs are mainly present in synovial fluid. Taking the Wiskott-Aldrich syndrome (WAS) as an example of DC dysfunction, it has been postulated that microenvironmental conditions in the RA joint might affect DC function, in that DCs could activate T cells in an inappropriate (uncontrolled) manner. This type of activation could occur through the engagement of surface molecules such as CD98 and CD147, since CD147 is expressed in synovial cells (including $T$ cells).

Systemic lupus erythematosus (SLE) is often described as a Th2 inflammatory disease, although blocking IFN $\gamma$ has been useful in animal models [3]. Dr E Liew (Department of Immunology, University of Glasgow, UK) demonstrates that injection of IL-18 with or without IL-12 induces SLElike symptoms in MRL/lpr mice (proteinurea, glomeru- 
lonephritis, and butterfly-like skin lesion around the muzzle). Although the cytokine profile depends on the genetic background, IL-18 injected alone induces the Th2 profile, whereas IL-18 and IL-12 injection induces the Th1 profile. This suggests that SLE might involve both Th1 and Th2 cells.

Dr I Mclnnes introduced the renal glomerulus as a model for studying cell contact interactions. Indeed, in the mesangium, $\mathrm{T}$ cells are in contact with epithelial cells. T cells stimulated with PMA (phorbol myristate acetate) induce the production of chemokines (MCP-1, RANTES, IP-10) in epithelial cells in a contact-dependent manner. This is mainly due to membrane-associated cytokines. In glomerulonephritis, contact between infiltrating cells induces ICAM-1 (intercellular adhesion molecule 1) and IL-6 expression but not MCP-1 production in mesangial cells. This profile is due to contact between mesangial cells and macrophages through the engagement of CD40 and is enhanced in the presence of TNF- $\alpha$ and IL-1 $\beta$. Another system for studying cell-cell interactions in RA was presented by $\mathrm{Dr} \mathrm{P}$ Life (Neurology and Rheumatology Systems, GlaxoWellcome Research and Development, Stevenage, UK), who adapted the Stamper Woodruff adhesion assay for use with cryostat tissue sections of RA synovium or tonsil over which target cells can be incubated in order to address the question of cytokine production 'in situ'. Results show that in such a culture system IL-1 $\beta$ production can be induced in the monocytic cell line THP-1.

Another way of interfering with immune cell infiltration is to hamper their interaction with endothelial cells. $\operatorname{Dr} A$ Hamann (Experimental Rheumatologie, Charite and Rheumaforschungszentrum, Berlin, Germany) showed that inhibitors of $\alpha 4$ integrins suppress collagen-induced arthritis in mice, although to a lower extent than steroids. Whether this effect is only due to blocking of the recruitment of leukocytes into the tissue remains to be determined. However, since this type of treatment in mice mobilizes hematopoietic precursors cells to peripheral blood, but does not lead to a depletion of these cells within bone marrow, it might be proposed for RA treatment.

\section{T cell anergy in RA}

A possible involvement of the jagged/notch genes, which are differentially induced in RA synovium, was discussed (Drs F Ponchel and M Ali, Molecular Medicine Unit and Department of Rheumatology, The University of Leeds, Leeds, UK). These genes are implicated in mechanisms involved in T-cell differentiation and thus might be involved in T-cell anergy. Whether T cells in RA synovium are anergic has been proposed, challenged and seriously discussed by many workers. Indeed, RA T cells display some anergic features (eg the lack of IL-2 production, stagnation at the G1 stage of the cell cycle, anergic-type $\mathrm{Ca}^{2+}$ fluxes) but as they are still mediating functions, they cannot be defined as anergic. This pseudo-anergy may be induced by direct cellcell contact of T cells with fibroblast-like synoviocytes which display an HLA-DR+, CD80- phenotype (Dr G Panayi, Rheumatology Unit, Guy's Hospital, London, UK). Indeed, T cells in RA are CD69+, CD25+, HLA-DR+, and VLA-1+, they produce small amounts of type 1 cytokines, are poorly proliferating, are arrested in the G1 stage of the cell cycle, but are CD45RO+. This is mainly due to contact with fibroblast-like synoviocytes which induce an upregulation of CD69 and CD25 in T cells in the absence of cell proliferation. The premise that fibroblast-like synoviocytes transfected with CD80 triggered T-cell proliferation suggests that fibroblast-like synoviocytes induce T-cell 'anergy' because they lack CD80 expression.

\section{Synoviocytes and bone}

The maintenance of bone mass is due to the tight regulation of osteoclast and osteoblast functions, which originate from hematopoietic and stromal cells, respectively (Dr G Russell, Department of Human Metabolic and Clinical Biochemistry, University of Sheffield Medical School, Sheffield, UK). In osteopetrosis, adipocytes accumulate instead of osteoclasts. Since these two types of cell originate from the same precursor cell, the accumulation of the wrong cell might be due to the lack of, or the overexpression of (a) differentiation factor(s). Since leptin-deficient mice have enhanced bone mass, leptin might be involved in this regulatory system. On the other hand, the differentiation of precursor cells into osteoclasts depends on the interaction of ODF and RANK (receptor-activator of NFKB), which are members of the TNF and TNF receptor family, respectively. A defect in this type of factor may lead to osteopetrosis. In contrast, overexpression of such a factor might lead to bone resorption. Therefore, action on this kind of factor might diminish bone erosion in rheumatic diseases.

The question of factors that trigger osteoclasts to digest bone during inflammatory conditions was addressed by $\mathrm{Dr}$ $S$ Goldring (Division of Rheumatology and Metabolic Bone Diseases, Beth Israel Deaconess Medical Center, Boston, MA, USA). In RA, tartrate-resistant acid phosphatase (TRAP), a marker of osteoclasts, is expressed by cells at the surface of the bone. This suggests that osteoclasts are indeed present in RA and might be responsible for bone erosion. Osteoclast maturation is regulated by ODF, which binds to RANK. ODF is produced by both osteoblastic cells and activated T cells; the latter cells might be involved in the imbalance of function of osteoclasts and osteoblasts in RA. This suggests that the ODF decoy receptor OPG might be a therapeutic means of diminishing bone erosion in RA.

\section{Conclusions}

The meeting shed light on several points that are now consensual: (i) in noninfectious conditions such as RA, the 
induction of proinflammatory cytokines in monocytemacrophages is mainly triggered by cellular contact interactions with stimulated $\mathrm{T}$ lymphocytes; (ii) the type of T-cell stimulus controls the choice of the signaling pathway in monocyte-macrophages, leading to either 'controlled' or 'uncontrolled' inflammatory processes; and (iii) several loops maintain proinflammatory and prodestructive mechanisms. Therapeutic approaches could be directed to these mechanisms in the future.

\section{References}

1. Firestein GS, Zvaifler NJ: How important are $T$ cells in chronic rheumatoid synovitis? Arthritis Rheum 1990, 33:768-73.

2. Nanki T, Lipsky PE: Cytokine, activation marker, and chemokine receptor expression by individual CD4+ memory $\mathrm{T}$ cells in rheumatoid arthritis synovium. Arthritis Res 2000, 2:415-423.

3. Ozmen L, Roman D, Fountoulakis M, Schmid G, Ryffel B, Garotta G: Experimental therapy of systemic lupus erythematosus: the treatment of NZB/W mice with mouse soluble interferon-gamma receptor inhibits the onset of glomerulonephritis. Eur J Immunol 1995, 25:6-12.

Author's affiliation: Clinical Immunology Unit, University Hospital, Geneva, Switzerland

Correspondence: Danielle Burger, Clinical Immunology Unit, Division of Immunology and Allergy, University Hospital, 1211 Geneva 14, Switzerland. Tel: +41 22372 9376; fax +41 22372 9369;

e-mail: danielle.burger@hcuge.ch 